Paper

\title{
Network structure reconstruction using packets of spikes in cultured neuronal networks coupled to microelectrode
} arrays

\author{
Timothée Leleu ${ }^{1 a)}$, Timothée Levi ${ }^{1}$, Takashi Kohno ${ }^{1}$, \\ and Kazuyuki Aihara ${ }^{1}$ \\ ${ }^{1}$ Institute of Industrial Science, The University of Tokyo, \\ 4-6-1 Komaba, Meguro-ku, Tokyo 153-8505, Japan. \\ a) timothee@sat.t.u-tokyo.ac.jp
}

Received July 31, 2017; Revised December 1, 2017; Published April 1, 2018

\begin{abstract}
Reconstructing accurately the structure of neural networks from biological data is essential for the analysis of simultaneous recordings from many neurons, and, in turn, for the understanding of neural codes and the design of neural prostheses. Classical techniques are generally based on cross-correlations and cannot reconstruct unambiguously the network structure. Recently, we have proposed a method for which there is one-to-one correspondence between statistical properties of packets of spikes (or avalanches) and the network structure, but this mapping was only proven for simpler neuronal model. In the following, we show using numerical simulation of the Izhikevich model that the proposed method is general, and is particularly well-fitted for the analysis of neural activity recorded from cultured neuronal networks coupled to microelectrode arrays.
\end{abstract}

Key Words: network reconstruction, spike analysis, avalanches, cultured neural networks, microelectrode arrays

\section{Introduction}

Neurological disorders affect millions of people around the world which calls for the development of neural prostheses that could restore cognitive and/or motor capabilities. The realization of prostheses requires understanding the connection between cells in the biological tissue and constructing connections between cells and the non-organic machine. In order to engineer the interface between neuronal cell assemblies and the prosthesis, it is necessary to take into account the intrinsic spontaneous activity of neuronal networks and to understand how to drive them into a desired state or to produce a specific behavior $[1,2]$. The accurate simulation of neuronal activity has become possible using biologically realistic neuron models that can reproduce numerous electrophysiological patterns recorded experimentally at the level of single cells at a low computational cost [3]. In addition, the long-term goal of replacing damaged brain areas with artificial devices also requires knowing the 
neural network structure, in particular the details of the micro-connectivity such as the strength of synaptic connections between pairs of neurons.

"In vitro" cultured neuronal networks coupled to microelectrode arrays (MEAs) constitute a valuable experimental model for studying the initial structure of a neural network and changes in the neuronal dynamics at different stages of development of the neural tissue. The main advantage of "in vitro" cultures is their relatively low noise level compared to "in vivo" experiments, which is due to smaller synaptic density and better controlled experimental environment. Being able to control the interactions between the different cells is a definite advantage for studying accurately the underlying neural code. However, even for "in vitro" experiments, the anatomical connectivity is not known or can be only partially estimated, therefore, efficient techniques for inferring the network structure from recorded activity would be a helpful tool for understanding network interactions.

Although experimental techniques are rapidly becoming more accurate and allow recording a greater number of neurons simultaneously, solving the network reconstruction inverse problem also requires from the analytical viewpoint to infer the most likely network structure from the experimental data. In the ideal case, an unambiguous reconstruction of the real network structure would be possible if there exists a one-to-one correspondence between the recorded data and the synaptic connection strength matrix. However, it is well-known that classical reconstruction methods, such as pairwise correlations [4], are not sufficient for the unambiguous reconstruction of the network structure [5]. Cross-correlations between a pair of neurons for example can either be interpreted as the result of connections between elements of the pair or of the effect of a shared input [5]. Moreover, crosscorrelation techniques are not adapted for determining the directionality of connections, which must be inferred using complementary methods, such as the calculation of information transfer [6]. Other methods have used information about spike trains [7] or the phase of coupled oscillators [8,9], and require the response to external perturbations [7-9].

Recently, it has been proposed to utilize the higher order statistics of spike trains in order to reconstruct the network structure unambiguously $[10,11]$. The occurrence of higher order correlations in neuronal activity has been notably revealed by the observation that the brain operates near a critical state [12-14]. In this regime, the cascading of neuronal activity is characterized by a branching ratio, i.e., the average number of neurons firing in response to one action potential, that is close to 1 when the system is near critical. Consequently, the occurrence of a single action potential can trigger the cascading of activity in the network, characterized by stereotypical spatio-temporal patterns $[15,16]$. This cascading of neuronal activity is called neuronal avalanche [12]. Although there are evidences that neuronal avalanches occur both "in vitro" $[12,13]$ and "in vivo" $[17,18]$, the avalanches can be identified particularly clearly "in vitro" for which the background activity is less noisy and does not interfere with the packets of spikes that result from intra-network connectivity. In [11], it has been shown that an unambiguous network structure can be associated to the statistical properties of neuronal avalanches and a one-to-one relation has been proven in the case of integrate-and-fire (IF) neuronal model. Moreover, it has been shown that the network structure can be accurately reconstructed from the analysis of neuronal avalanches even when the effect of the leak is taken into account, i.e., using the leaky IF model [11]. In the following, we consider the generalization of this method to more realistic spiking neuron models. We show using numerical simulations of Izhikevich spiking neurons [3] and analytical arguments that the proposed method can in fact be applied to various models and allows for a more accurate reconstruction of the network structure than classical methods. We argue that the proposed method is well-fitted for the analysis of neural activity recorded from cultured neuronal networks coupled to microelectrode arrays.

\section{Classic network reconstruction methods}

In the following, we explain the methodology used for achieving network reconstruction from neural activity of cultured cells coupled to microelectrode arrays. We first describe the pre-processing of the raw data. Second, we review classical methods used for the analysis of the spike trains. Note that we limit the scope of the methods to the reconstruction of excitatory connections for the sake of 


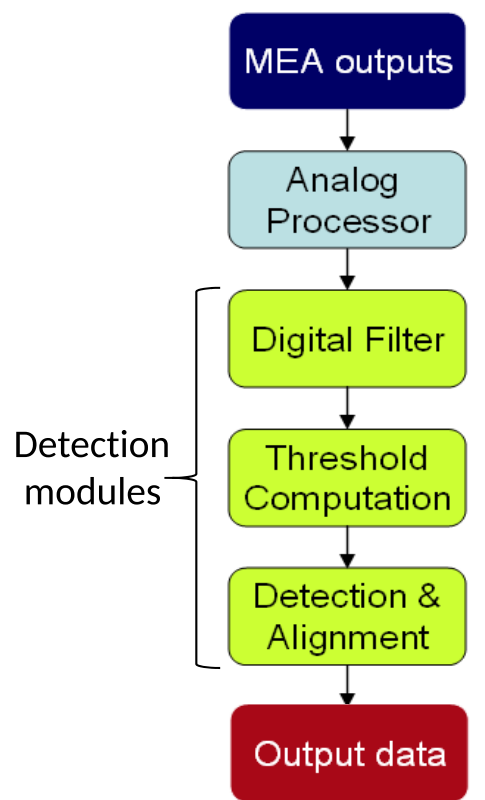

Fig. 1. Processing chain for spike detection. Analog processor is a low-noise amplifier. The green modules are digital and are composed of digital filter for removing DC signal and LFP (Low Field Potential), threshold computation for evaluating the noise, detection \& alignment for detector, and extraction of the spikes.

simplicity. We note $\Omega=\left\{\omega_{i j}\right\}_{i j}$ the matrix of anatomical synaptic connections and $\tilde{\Omega}=\left\{\tilde{\omega}_{i j}\right\}_{i j}$ the inferred network structure. The number of excitatory neurons is noted $N_{\mathrm{e}}$.

\subsection{Pre-processing of raw data}

The first step of the reconstruction consists in the detection of spikes from the recorded biological data. Extracellular spikes from "in vitro" culture have typically weak amplitude signals $(50-500 \mu V)$, a spike frequency in the $100-\mathrm{Hz}$ to $10-\mathrm{kHz}$ frequency band, and their duration is a few milliseconds. For active neurons, the spiking rate usually varies from 10 to 120 occurrences per second. With these characteristics, visual spike detection becomes ineffective and real-time spike detection is essential. To make the detection easier and reduce noise, the recorded raw data is processed with amplifiers and filters. Then the detection algorithm, which can be based on wavelet transform [19], is applied. In a nutshell, the sub-steps of the spike detection are the following: (1) bandwidth reduction for selective band amplification and noise reduction, (2) discrimination threshold computation, (3) extraction and alignment of neurobiological spike signals [20,21] (see Fig. 1). Once the spikes have been identified, the analysis of spike trains can be used to reconstruct the network structure.

\subsection{Binning of spiking activity}

The second step consists in the time-binning of the spiking activity. Given a spike train $\left\{t_{j}^{k}\right\}_{j, k}$ of $N_{\mathrm{e}}$ isolated neurons, where $t_{j}^{k}$ is the $k^{t h}$ time when the neuron $j$ spikes with $j \in\left\{1, \cdots, N_{\mathrm{e}}\right\}$, the binary matrix $A$ represents the time-binning of this spiking activity as follows:

$$
A(n, j)=\left\{\begin{array}{l}
1 \text { if } \exists k \mid t_{j}^{k} \in\left[t_{0}+n \Delta t, t_{0}+(n+1) \Delta t\right], \\
0 \text { otherwise }
\end{array}\right.
$$

where $\Delta t$ is the time-bin duration and $t_{0}$ the starting time of the experiment. Note that if $\Delta t$ is taken sufficiently small ${ }^{1}$, there is only one spike per time-bin.

\subsection{Pairwise correlations}

The network structure is classically reconstructed using the correlation matrix of spiking activity [4, 22]. This method infers the functional rather than anatomical connectivity. Generally, we can define

\footnotetext{
${ }^{1}$ Here, sufficiently small means smaller than the minimal interspike interval of the spike train $\left\{t_{j}^{k}\right\}_{k}, \forall j$.
} 
the correlation $c_{i j}$ of spiking activity within packets between neurons $i$ and $j$ as follows $((i, j) \in$ $\left.\left\{1, \cdots, N_{\mathrm{e}}\right\}^{2}\right)$ :

$$
c_{i j}=\frac{\left.<\delta \nu_{i}(t) \delta \nu_{j}(t)\right\rangle_{t}}{\sqrt{\left.<\delta \nu_{i}(t)^{2}\right\rangle_{t}} \sqrt{\left.<\delta \nu_{j}(t)^{2}\right\rangle_{t}}},
$$

where $\left\langle>_{t}\right.$ represent time averages; $\nu_{i}(t)$, the instantaneous firing rate obtained by smoothing the spike train over a window of size $1 \mathrm{~ms}(\approx$ average duration of spike packets), at proximity of the time $t ; \delta \nu_{i}(t)$, the variation of firing rate given as $\delta \nu_{i}(t)=\nu_{i}(t)-\left\langle\nu_{i}(t)\right\rangle_{t}$. The network structure is estimated using the pairwise correlations as follows:

$$
\tilde{\omega}_{i j} \propto c_{i j}
$$

\subsection{Renormalized pairwise correlations}

We propose another method that utilizes the raw temporal correlations between the variations $\phi\left(\nu_{i}(n)\right)$ of the firing rates estimated at proximity of a time-bin $n$, obtained after renormalization of firingrate variations using the mean and standard deviations calculated among neurons, i.e., $\phi(X)=$ $\frac{X-\mu_{X}}{\sigma_{X}}$ where $\mu_{X}$ and $\sigma_{X}$ are the mean and standard deviation of the distribution of the variable $X$, respectively, with $\mu_{\nu}=\frac{1}{N_{\mathrm{e}}} \sum_{i=1}^{N_{\mathrm{e}}} \nu_{i}(n)$ and $\sigma_{\nu}^{2}=\frac{1}{N_{\mathrm{e}}} \sum_{i=1}^{N_{\mathrm{e}}}\left(\nu_{i}(n)-\mu_{\nu}\right)^{2}$. Moreover, $\nu_{i}(n)$ is the average number of spikes from the neuron $i$ per time-bin calculated over a window of length $\Delta$ time-bins, i.e., $\nu_{i}(n)=\frac{1}{\Delta} \sum_{k=0}^{\Delta} A(n+k, i)$. We set $\Delta$ to $\Delta=400$ time-bins in numerical simulations. The raw temporal correlations are obtained by taking the temporal average among time-bins $n$ as follows:

$$
c_{i j}^{\prime}=<\phi\left(\nu_{i}(n)\right) \phi\left(\nu_{j}(n)\right)>_{n},
$$

with $<>_{n}$ the temporal averages over the time-bins indexed by $n$. This method allows taking into account correlations between neurons only if they spike more than other neurons do within the timebin of length $\Delta$. This method also allows reducing the effect of temporal variations of the population averaged activity on the calculation of correlations. The network structure is estimated using the time-averaged correlation as follows:

$$
\tilde{\omega}_{i j} \propto c_{i j}^{\prime}
$$

\subsection{Consecutive time-bins}

The pairwise correlation method described in Eqs. (2) and (3) cannot be used to detect the direction of connections. In order to infer directionality, we consider another method that uses the spiking of activity in two consecutive time-bins. First, we detect the time-bins $n$ in which: the neuron $j$ fires a spike while no spikes were detected in the previous time-bin $n-1$. Then, if another neuron $i$ also fired in response to the activity of neuron $j$ at the time-bin $n+1$, we record this activity by noting $\pi_{i j}^{(p)}=1$ and $\pi_{k l}^{(p)}=0$ for $(k, l) \neq(i, j)$ with $p$ the index of the $p$ th pair of sequential spiking activity recorded. We define the probability that the neuron $j$ triggers the neuron $i$ as follows:

$$
\pi_{i j}=\frac{1}{N_{j}} \sum_{p} \pi_{i j}^{(p)},
$$

where $N_{j}$ is the number of avalanches that are triggered by the neuron $j$. The network structure can then be inferred by making the following assumption:

$$
\tilde{\Omega} \propto \Pi,
$$

with $\Pi=\left\{\pi_{i j}\right\}_{i j}$. In other words, the inferred strength of synaptic connection from neuron $j$ to $i$ is assumed to be proportional to the probability that the neuron $i$ fires in response to neuron $j$, given that no other neurons were active prior to $j$. 


\section{Proposed method}

\subsection{Bursts of action potentials}

The hypothesis of Eq. (7) is justified if the duration of the time-bins $\Delta t$ corresponds to the synaptic delay between two neurons. In this case, sequential firing between two neurons can only result from direct anatomical connections because we have considered only time-bins in which no neurons fired prior to the triggering neuron. However, the uncertainty about synaptic delays make this method not applicable to the analysis of real data, in which sequential firing can either result from direct anatomical connections, or indirect paths that involve the activity of intermediate neurons. In order to take into account all possible indirect paths, the method that we propose uses longer packets of spiking activity.

A packet, indexed by $p$ and starting at the time-bin $n_{p}$, is defined as the spiking activity $A_{p}$ in subsequent time-bins that is preceded and succeeded by two empty time-bins indexed by $n_{p}-1$ and $n_{p}+\Delta n_{p}$, respectively, where $\Delta n_{p}$ is the duration of the packet $p$. The duration of a packet can be longer than 2 time-bins, so that this method is a generalization of the one using only two consecutive time-bins. The definition of packets is illustrated in Fig. 2.

The spiking activity during a packet $A_{p}$ is given as follows:

$$
A_{p}=\{A(n, i)\} \underset{n \in\left\{n_{p}, \cdots, n_{p}+\Delta n_{p}-1\right\}}{(n, i)}
$$

given the following conditions:

$$
\begin{array}{r}
\exists i \mid A(n, i) \neq 0 \text { for } n \in\left\{n_{p}, \cdots, n_{p}+\Delta n_{p}-1\right\}, \\
A\left(n_{p}-1, i\right)=A\left(n_{p}+\Delta n_{p}, i\right)=0, \forall i .
\end{array}
$$

The spiking activity during a packet $p$ can be summarized by the activity vector $\boldsymbol{L}_{j}^{(p)}$ defined as $\boldsymbol{L}_{j}^{(p)}=\left\{L_{i j}^{(p)}\right\}_{i}$ and $L_{i j}^{(p)}=\sum_{n=n_{p}}^{n_{p}+\Delta n_{p}-1} A(n, i)$ with $j$ the index of the neuron that has triggered the packet $p$, i.e., $j(p)=\left\{i \mid A\left(n_{p}, i\right) \neq 0\right\}$, if there is a single neuron spiking in the time-bin indexed by $n_{p}$. Note that $j$ can always be defined uniquely if the time-bin $\Delta t$ is sufficiently small.

Lastly, we define the averaged composition of packets by distinguishing the activity of neurons $i$ during packets as a function of the neurons $j$ that have triggered them. The averaged composition of packets that starts from the neuron $j$ is noted $m_{i j}$ and is defined as follows:

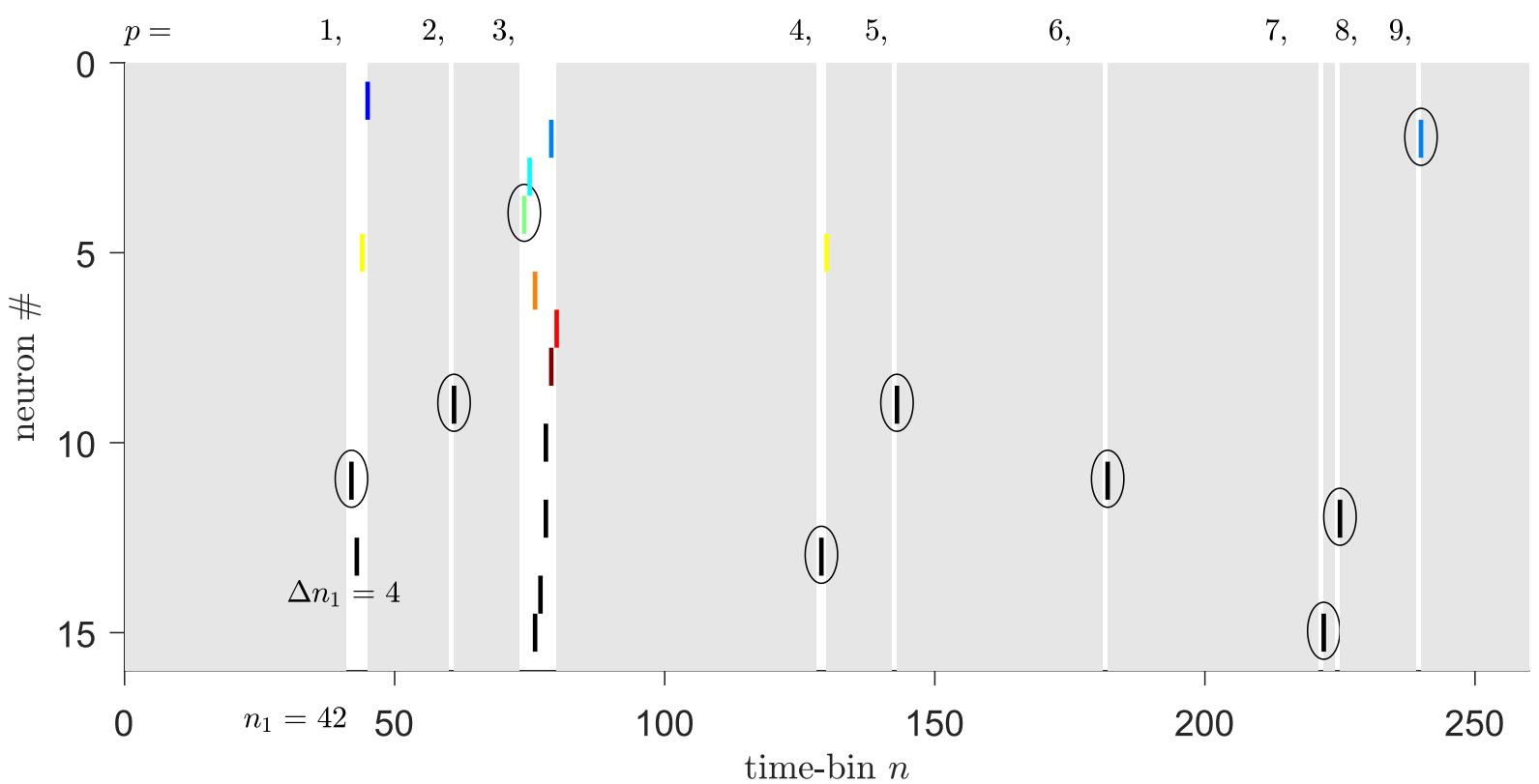

Fig. 2. Raster plot of spiking activity and illustration of activity packets. Time-bins during which no spikes occur are colored in gray, and white intervals represent packets. Packets are indexed from $p=1$ to $p=9$ and the first spikes that trigger activity packets are circled. $\Delta t=0.05 \mathrm{~ms}$. 


$$
m_{i j}=\frac{1}{N_{j}} \sum_{p} L_{i j}^{(p)}
$$

where $N_{j}$ is the number of packets that start from the neuron $j$. If the neurons spike at most once per packet, the value of $m_{i j}$ can also be interpreted as the probability that the neuron $i$ spikes during a packet that is triggered by the neuron $j$. Note that the synchrony of spikes is related to the size of packets: larger packets are a signature of greater synchronous firing.

In practice, it is possible that two neurons fire at the first time-bin of a packet, in which case distinguishing the triggering neuron of this packet becomes ambiguous. We resolve this ambiguity by allowing a given packet to have several triggering neurons $j$ when calculating the elements $m_{i j}$ in Eq. (11). Numerical simulations (see section 4) show that the proposed method remains accurate even when the probability of having multiple triggering neurons is close to $30 \%$.

Given the averaged composition matrix of packets $M=\left\{m_{i j}\right\}_{i j}$ that is calculated using only the action potentials of excitatory neurons, the network structure can be inferred using the following formula, called inversion formula [11]:

$$
\tilde{\Omega} \propto I-M^{-1},
$$

where $I$ is the identity matrix of size $N_{\mathrm{e}} \times N_{\mathrm{e}}$ with $N_{\mathrm{e}}$ the number of excitatory neurons and $\tilde{\Omega}=\left\{\tilde{\omega}_{i j}\right\}_{i j}$ the inferred synaptic matrix weights from excitatory to excitatory neurons. Note that Eq. (12) requires $M$ to be invertible, i.e., that $\operatorname{det}(M) \neq 0$. Also, Eq. (12) implies that $M=(I-\tilde{\Omega})^{-1}$ which can be rewritten as follows using the Neumann series in $\tilde{\Omega}$ :

$$
M=\sum_{n=0}^{\infty}(\tilde{\Omega})^{n} .
$$

\subsection{Interpretation of the inversion formula}

The averaged composition matrix formula given in Eq. (12) is exact in the case of integrate-and-fire neuron models. In order to show this, we have recently extended the model of Eurich et al. [24] that is equivalent to a non-leaky IF model when the number of neurons is large and derived the probability distribution of spike packets [11]. Using the probability distribution, the averaged composition matrix can be derived without approximations and is given as described in Eq. (12).

Although the formula has only been proven in the case of IF neurons, its interpretation is general and can be applied to other spiking neuron models. We first consider the case of two isolated neurons. We assume that the probability that a neuron $j$ triggers the spiking of the neuron $i$ in the next time-bin is proportional ${ }^{2}$ to $\omega_{i j}$. In the case of three isolated neurons, the neuron $j$ can either trigger directly the neuron $i$ with probability $\omega_{i j}$, or the neuron $j$ can trigger the neuron $k$ which, in turn, triggers the neuron $i$. Thus, the total probability that a neuron $j$ triggers a neuron $i$ can be written $\omega_{i j}+\omega_{j k} \omega_{k i}$. In the case of $N_{\mathrm{e}}$ neurons, we can generalize the previous arguments. The probability that a neuron $j$ triggers the spiking of the neuron $i$, noted $m_{i j}$, is then given as $\omega_{i j}+\sum_{k} \omega_{j k} \omega_{k i}+\sum_{k l} \omega_{j k} \omega_{k l} \omega_{l i}+\cdots$. In the matrix form, $\left\{m_{i j}\right\}_{i j}$ is thus described as in Eq. (13).

\subsection{Importance of higher-order correlations}

The interpretation of the inversion formula suggests that the higher-order terms $(\Omega)^{n}$ correspond to the spiking activity that occurs from the $n$th time-bin after the spike-time of the triggering neuron. Thus, the truncation of the formula at the order $K$ allows for an approximate estimation of the average packet sizes $M^{(K)}$ that do not take into account spikes occurring after the $K$ th bin as follows:

$$
M^{(K)}=\sum_{n=0}^{K}(\Omega)^{n},
$$

with $M^{(K)} \rightarrow M$ when $K \rightarrow \infty$.

\footnotetext{
${ }^{2}$ Of course, the probability that a neuron $j$ triggers the spiking of the neuron $i$ in the next time-bin is generally a non-linear function of $\omega_{i j}$. When $\omega_{i j} \ll 1$, we can however approximate this probability by the linearization of this non-linear function.
} 

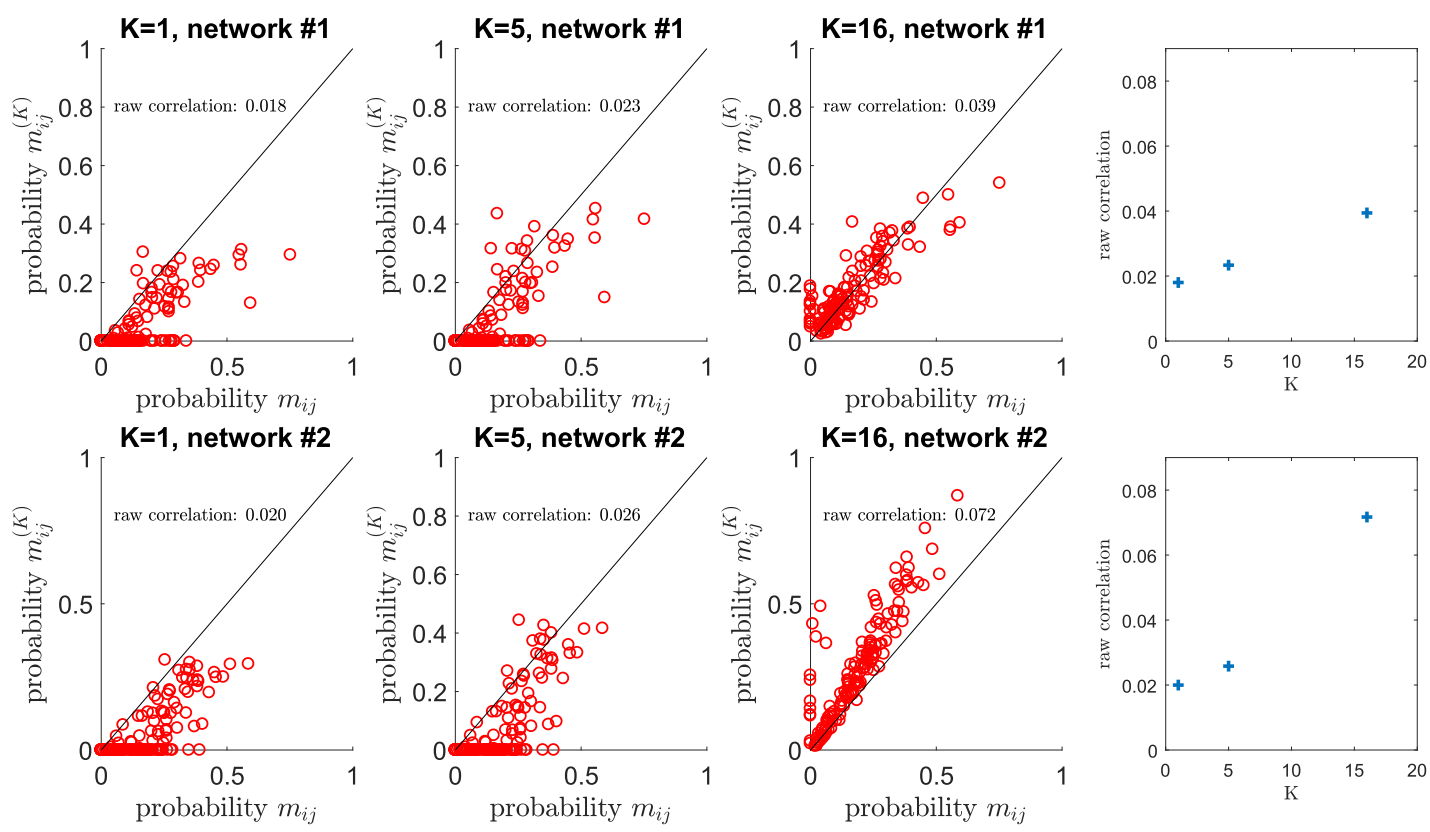

Fig. 3. Probability $m_{i j}$ that a neuron $i$ fires during a packet triggered by the neuron $j$ calculated using numerical simulation of a network of $N_{\mathrm{e}}=12$ excitatory IF neurons vs. estimated probability $m_{i j}^{(K)}$ using the Eq. (14), respectively. In each panel is shown the raw correlation between $m_{i j}$ and $m_{i j}^{(K)}$ given as $\frac{1}{N_{\mathrm{e}}\left(N_{\mathrm{e}}-1\right)} \sum_{i j(i \neq j)} m_{i j} m_{i j}^{(K)}$, for $K \in\{1,5,16\}$. Raws depict the results for two different network structure $\Omega$, in which synaptic weights are drawn from uniform distribution with a sparsity of $40 \%$. The last column on the right shows the raw correlations vs. $K$.

(a)

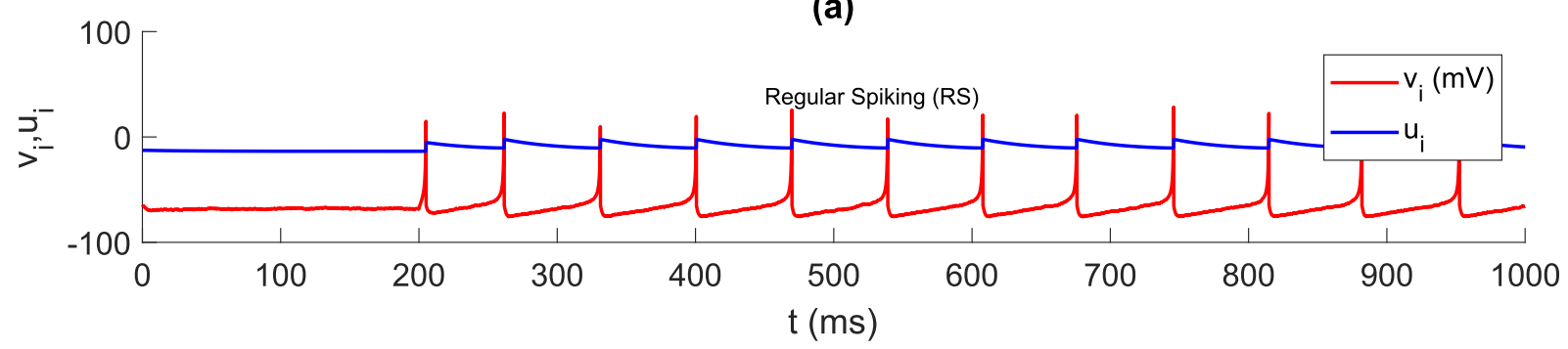

(b)

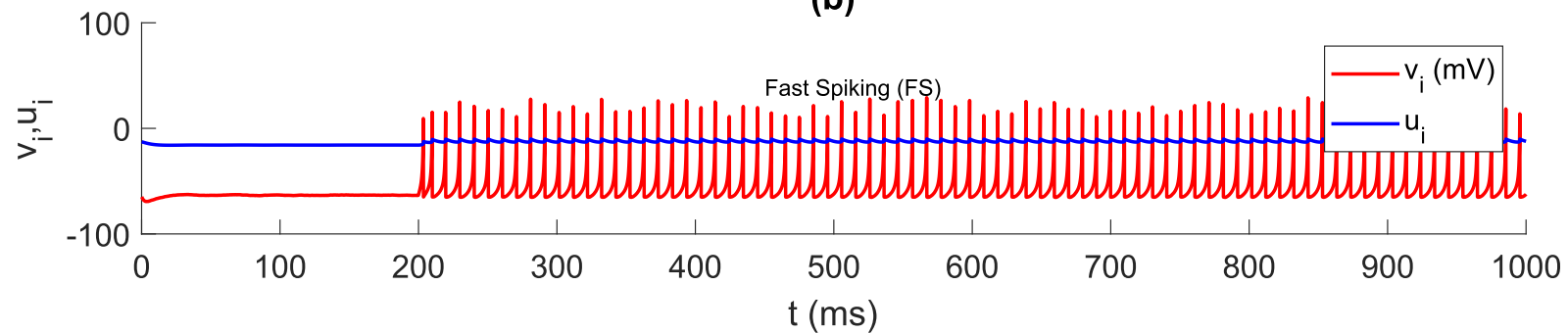

(c)

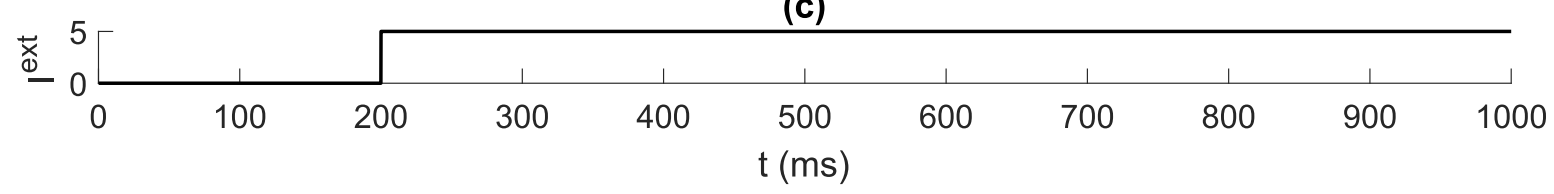

Fig. 4. Typical time series of the Izhikevich model. (a) and (b) show time series of the variables $v_{i}$ and $u_{i}$ for regular and fast spiking neurons, respectively, when the external input $I^{\text {ext }}$ is given as shown in (c). The former and latter models are used for the simulation of excitatory and inhibitory neurons, respectively (see Table B-I for the corresponding parameters). 
(a)
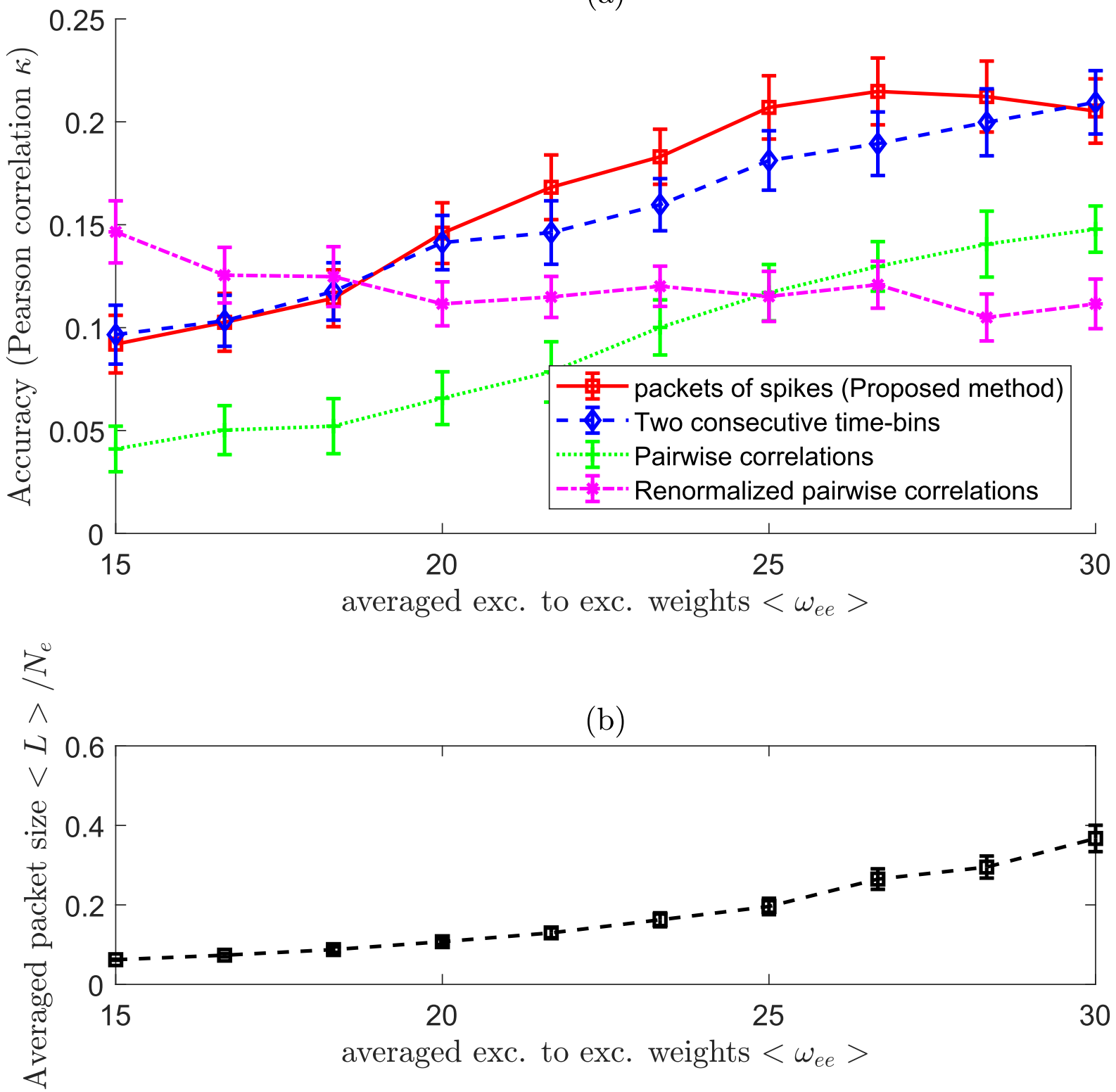

Fig. 5. (a) Accuracy of the network reconstruction measured by the Pearson $\kappa$ correlation between inferred and true weights vs. averaged strength of excitatory to excitatory connections $\left\langle\omega_{e e}\right\rangle$. Full, dashed, and dotted, and dashdotted lines show the network reconstruction accuracy using the packet of spikes, two consecutive time-bins, pairwise-correlations, and renormalized pairwise correlations, respectively. (b) Averaged packet size $\frac{\langle L\rangle}{N_{\mathrm{e}}}$ vs. averaged strength of excitatory to excitatory connections $\left\langle\omega_{e e}\right\rangle$. Error bars represent the margin of error with a $95 \%$ confidence level using the assumption of normality over a sample of 50 trials. The duration of simulation is $T=50 \mathrm{~s}$ (50000 ms).

In the case $K=1$, Eq. (14) becomes equivalent to Eq. (7) with $\tilde{\Omega}=M^{(1)}-I$, i.e., the method that uses two consecutive time-bin is equivalent to using packets truncated at $K=1$. In order to illustrate the gain in accuracy that comes from the fact that longer packets are taken into account in the proposed method, we compare in Fig. 3 the probability $m_{i j}$ obtained using numerical simulation of a network of 12 excitatory neurons and the estimated probability $m_{i j}^{(K)}$ calculated using Eq. (14). Simulations in Fig. 3 are performed using the integrate-and-fire model. The calculation of correlations between $m_{i j}$ and $m_{i j}^{(K)}$ show that using only two consecutive time-bins ( $K=1$, left panels) does not give an accurate reconstruction of the probabilities $m_{i j}$. The accuracy in estimating $m_{i j}$ increases 
(a)

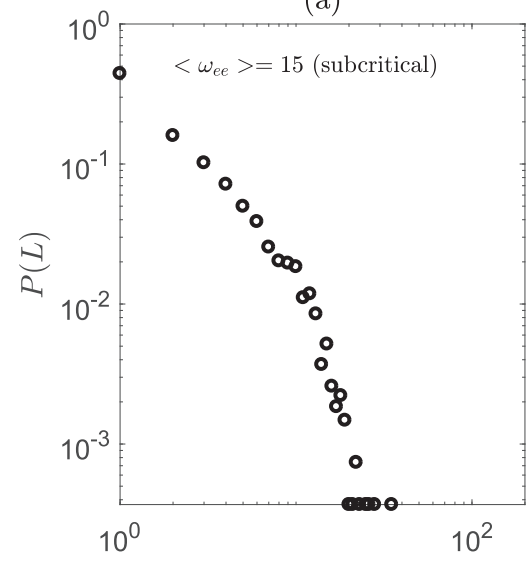

$L$

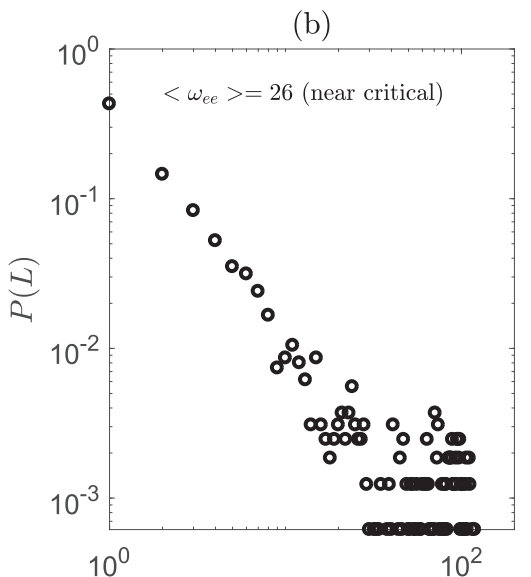

$L$

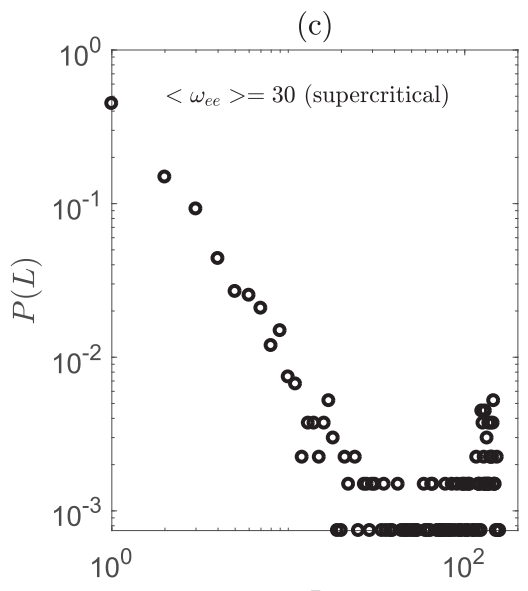

$L$

Fig. 6. (a), (b), and (c) Show the distribution $P(L)$ of avalanche sizes $L$ in the subcritical, near critical, and supercritical regimes, respectively.

progressively as $K$ increases (see Fig. 3 from left to right), such that it is necessary to take into account the cascading of spiking activity that occurs long after the time of the triggering neuron.

\section{Comparison of reconstruction accuracy using the Izhikevich model}

\subsection{Measure of network reconstruction accuracy}

In order to test the generality of the proposed method, we simulate the activity of neuronal networks using the Izhikevich model [3]. This model can reproduce numerous electrophysiological features of cortical neurons, such as spike frequency adaptation, bursting, resonance, and rebound spiking, which cannot be simulated using the integrate-and-fire neuron model. Such biomimetic neuron model is necessary for the development of next generation neuroprostheses [23]. The description of the model is given in appendix A, and the parameters used for numerical simulations in appendix B. The parameter values chosen for the numerical simulations of the Izhikevich model in the case of excitatory and inhibitory neurons correspond to regular spiking (RS) and fast spiking (FS) neurons, respectively. We utilize the same parameter values as given in [3] and add some variability to the parameter values for the sake of realism. Typical time series of these neuron models are shown in Fig. 4. Then, the accuracy of the network reconstruction methods are measured using the Pearson correlation $\kappa$ between inferred $\tilde{\omega}_{i j}$ and true weights $\omega_{i j}$ defined as follows:

$$
\kappa=\frac{1}{N_{\mathrm{e}}\left(N_{\mathrm{e}}-1\right)} \sum_{i j} \phi\left(\tilde{\omega}_{i j}\right) \phi\left(\omega_{i j}\right)
$$

with $\phi(X)=\frac{X-\mu_{X}}{\sigma_{X}}$ where $\mu_{X}$ and $\sigma_{X}$ are the mean and standard deviation of the distribution of the variable $X$, i.e., the set indexed by the indices $i$ and $j,(i, j) \in\left\{1, \cdots, N_{\mathrm{e}}\right\}^{2}$, in this case.

In order to get a better comparison between reconstruction methods, we set to zero negative values of $\tilde{\omega}_{i j}$ and keep fix fraction of weights $\tilde{\omega}_{i j}$ to be non-zero in all cases. This fraction is given by the sparsity of the original network structure. This avoids having larger reconstruction errors solely because a greater number of weights $\tilde{\omega}_{i j}$ are inferred to be non-zero for a given method.

\subsection{Accuracy vs. strength of excitatory to excitatory connections}

Figures 5(a) and (b) show that the proposed method performs better than the ones using the pairwise correlations and two consecutive time-bins when the size of the packets is intermediate, i.e., near the critical regime. The averaged packet size $\frac{<L>}{N_{\mathrm{e}}}$ is shown in Fig. 5(b), as a function of the averaged strength of excitatory to excitatory connections $<\omega_{e e}>$. On the one hand, smaller packets (subcritical regime) do not contain much information about the strengths of excitatory connections and the network structure cannot be interfered when spiking activity does not cascade in the network. On the 
(a)
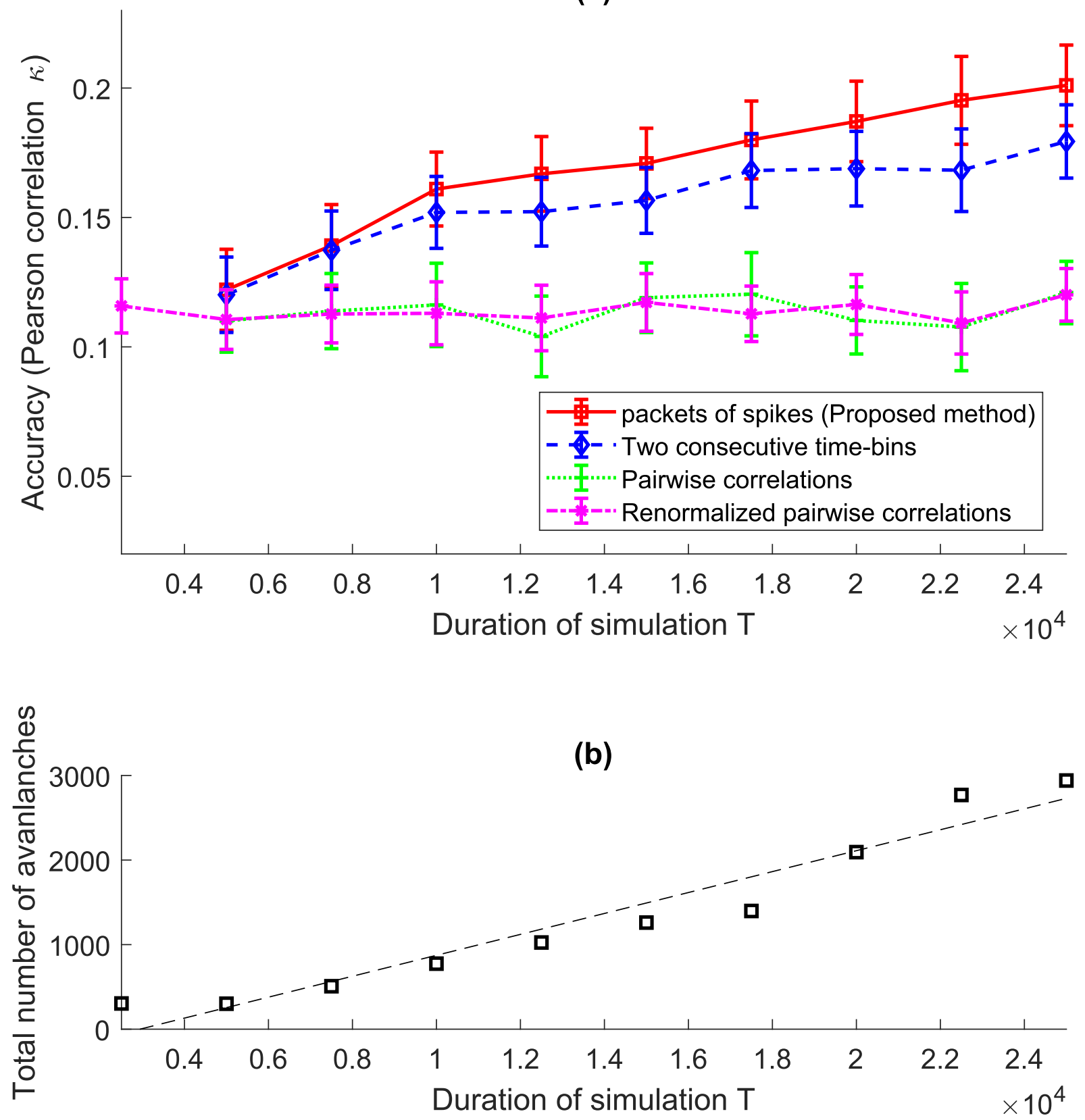

Fig. 7. (a) The same as in Fig. 5(a) but vs. the duration of simulation of simulation $T$. (b) Total number of avalanches vs. simulation duration $T$.

other hand, very large packets (supercritical regime) during which the whole network becomes active cannot be used to accurately infer the network structure because all packets become identical. In Fig. 6 is shown the distribution $P(L)$ of the avalanche sizes $L$ when the averaged recurrent excitatory connection strength $\left\langle\omega_{e e}\right\rangle$ is small, intermediate, and large which correspond to the subcritical, near critical, and supercritical cases, respectively.

The regime where packets are very small $\left(\frac{L}{N_{\mathrm{e}}} \approx 0\right)$ and very large $\left(\frac{L}{N_{\mathrm{e}}} \approx 1\right)$ in average correspond to subcritical and supercritical neuronal activity $[11,24]$. At the critical state, the distribution of sizes $L$ of avalanches is close to power law distribution. Note that the critical state is only defined in the limit $N \rightarrow \infty$, and the parameter regime that is the closest to the critical one in the case of smaller networks $(N<\infty)$ is usually displaced as compared to the case of the thermodynamical limit. Thus, Figs. 5 and 6 show that the proposed method is most accurate near the critical regime, 
(a)
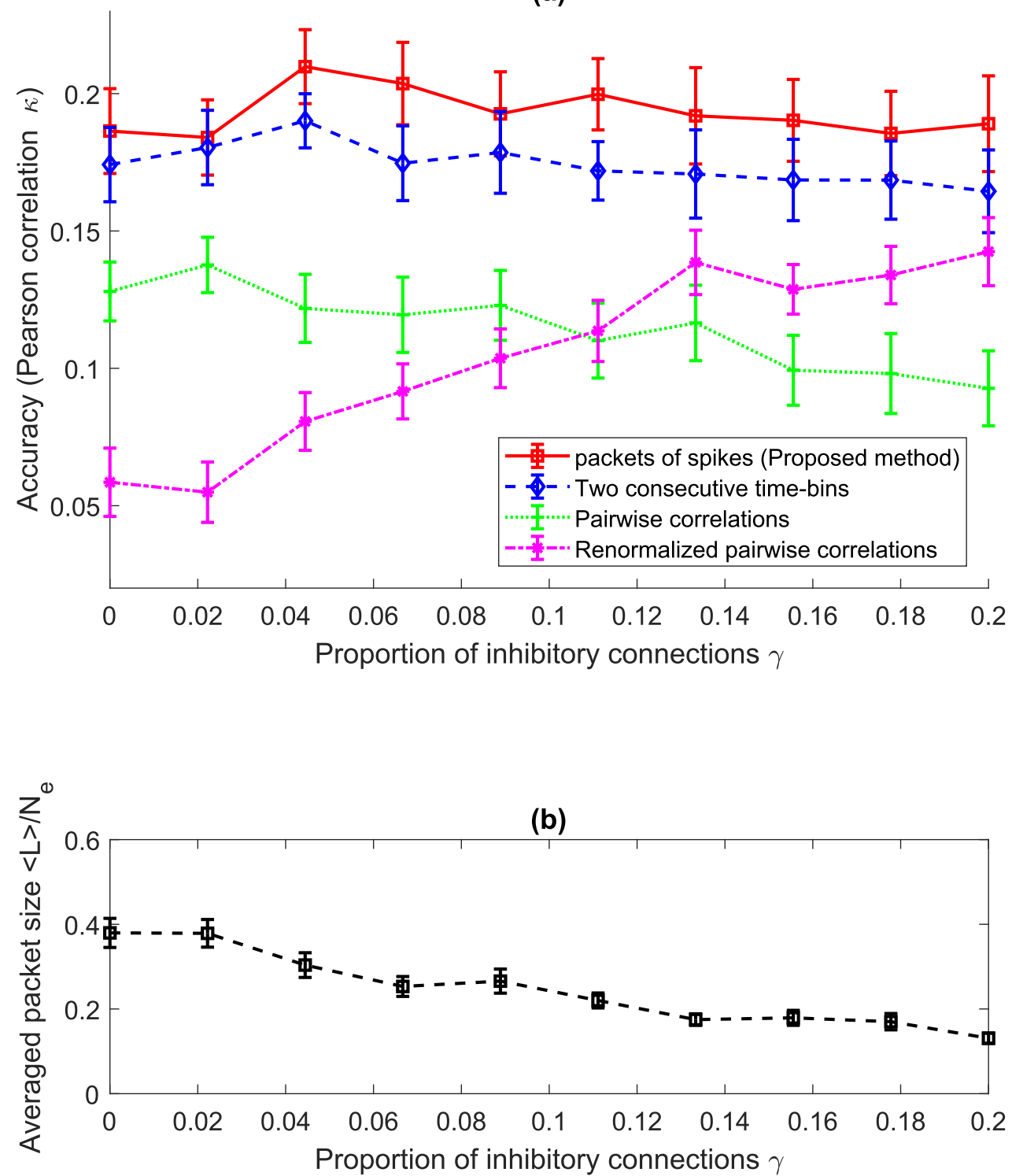

Fig. 8. (a) and (b) The same as in Figs. 5(a) and (b), respectively, but vs. the proportion of inhibitory neuron $\gamma$.

intermediate between the two extreme cases. The critical regime is expected to be the one at which neuronal networks operate $[12,13,24]$. Therefore, these results suggest that proposed method is the most suited for the analysis of biological data.

\subsection{Accuracy vs. duration of simulation}

Figure 7(b) shows that the proposed method requires more data, i.e., longer duration of simulations, in order to perform significantly better than the methods based on pairwise correlations. The accuracy of the latter methods do not improve after a certain duration of simulation, whereas the accuracy of the former increases steadily when using longer simulation duration.

\subsection{Accuracy vs. proportion of inhibitory neurons}

The proposed method can be used to infer the network structure of excitatory to excitatory connections, but do not take into account the effect of inhibitory neurons. Figure 8 shows that the method is more accurate than the other methods for realistic values of the proportion of inhibitory neurons, noted $\gamma$. 


\section{Conclusion}

In this paper, we have described a methodology for reconstructing the structure of synaptic connectivity between neurons of cultured neuronal networks coupled to microelectrode arrays. We have proposed a novel method for an accurate network reconstruction when the activity is near critical, which is the regime observed experimentally for developing cultured neuronal networks. By taking into account the higher-order correlations between spikes within packets, numerical simulations show that the proposed method allows for a gain in accuracy compared to classical methods.

The proposed method has been shown to be applicable to the network reconstruction of integrateand-fire [11], leaky integrate-and-fire [11], and Izhikevich spiking neural models (herein). In future works, the generality of the proposed method should be verified using other models, such as the DSSN model [25] or Hodgkin-Huxley model [26]. Moreover, we have not taken into account slower synaptic current dynamics, such as the ones induced by slow NMDA channels, nor the effect of short-term synaptic plasticity. Improvement in reconstruction accuracy of biological data may be achievable by taking into account these mechanisms. Lastly, the formula given in Eq. (13) can be adapted to fit a particular model. We are currently investigating the calculation of inversion formula that are tailored to each spiking neuron model. By incrementally increasing the complexity of the simulation model, we aim at the development of a robust methodology for the analysis and control of cultured neuronal networks.

\section{Acknowledgments}

This research was supported by ImPACT Program of Council for Science, Technology and Innovation (Cabinet Office,Government of Japan). This work was supported by NEC Corporation. We would like to thank the anonymous reviewers for their suggestions and comments.

\section{Appendix}

\section{A. Description of Izhikevich model}

Numerical simulations are performed using the simple Izhikevich model. The dynamics of the neuron $i$ is given as follows:

$$
\begin{aligned}
\partial_{t} v_{i} & =0.04 v_{i}^{2}+5 v_{i}+140-u+I_{i}^{e x t}+\sum_{k, j} \omega_{i j} \delta\left(t-t_{j}^{(k)}\right), \\
\partial_{t} u_{i} & =a\left(b v_{i}-u_{i}\right)
\end{aligned}
$$

where $v_{i}$ and $u_{i}$ represents the membrane potential and recovery variable of the neurons $i$, respectively, and $t_{k}^{(k)}$ is the time of the $k^{t h}$ spike triggered by the neurons $j$. When $v_{i}>30$, the neuron $i$ spikes and the variables $v_{i}$ and $u_{i}$ are reset to $c$ and $u_{i}+d$, respectively. Moreover, the synaptic noise is modeled by $I_{i}^{e x t}$ with $d I_{i}^{e x t}=\mu_{I} d t+\sigma_{I} d w_{i}$ and $d w_{i}$ the Wiener increment.

The weights $\omega_{i j}$ are drawn from uniform distribution with mean $\mu_{e e}=<\omega_{e e}>, \mu_{e i}=<\omega_{e i}>$, $\mu_{i e}=<\omega_{i e}>$, and $\mu_{i i}=<\omega_{i i}>$ for excitatory to excitatory, inhibitory to excitatory, excitatory to inhibitory, and inhibitory to inhibitory connections, respectively. Then, the weights $\omega_{i j}$ are set to zero with probability $\xi<1$, which creates a sparse network structure.

We consider networks with $N_{\mathrm{e}}$ and $N_{\mathrm{i}}$ excitatory and inhibitory neurons, respectively, with a fraction $\gamma<1$ of inhibitory ones, i.e., $\gamma=\frac{N_{\mathrm{i}}}{N_{\mathrm{e}}+N_{\mathrm{i}}}$. 


\section{B. Parameters for the Izhikevich model}

Table B-I. Simulation parameters.

\begin{tabular}{lll}
\hline \hline Notation & Detail & Value \\
\hline \hline$N_{\mathrm{e}}$ & number of excitatory neurons & 27 \\
$N_{\mathrm{i}}$ & number of inhibitory neurons & 3 \\
$\gamma$ & proportion of inhibitory neurons & 0.1 \\
$<\omega_{e e}>$ & mean excitatory to excitatory weights & 25 \\
$<\omega_{e i}>$ & mean inhibitory to excitatory weights & -2.5 \\
$<\omega_{i e}>$ & mean excitatory to inhibitory weights & 2.5 \\
$<\omega_{i i}>$ & mean inhibitory to inhibitory weights & -2.5 \\
$a$ & Izhikevich model: time-scale recovery (exc., inh.) & $0.02,0.02-0.1$ \\
$b$ & Izhikevich model: sensitivity (exc., inh.) & $0.2,0.20-0.25$ \\
$c$ & Izhikevich model: reset $v_{i}$ (exc., inh.) & $-65,-65$ \\
$d$ & Izhikevich model: reset $u_{i}$ (exc., inh.) & 8,2 \\
$\xi$ & network structure sparsity & 0.2 \\
$\Delta t$ & simulation time-step (width of time-bin) & 0.1 \\
\hline
\end{tabular}

\section{References}

[1] L.R. Hochberg, D. Bacher, B. Jarosiewicz, N.Y. Masse, J.D. Simeral, J. Vogel, S. Haddadin, J. Liu, S.S. Cash, P. van der Smagt, et al., "Reach and grasp by people with tetraplegia using a neurally controlled robotic arm," Nature, vol. 485, no. 7398, pp. 372-2012, May 2012.

[2] P. Bonifazi, F. Difato, P. Massobrio, G.L. Breschi, V. Pasquale, T. Levi, M. Goldin, Y. Bornat, M. Tedesco, M. Bisio, R. Galron, S. Kanner, J. Tessadori, S. Taverna, and M. Chiappalone, "In vitro large-scale experimental and theoretical studies for the realization of bi-directional brain-prostheses," Frontiers in Neural Circuits, vol. 7, p. 40, 2013.

[3] E.M. Izhikevich, "Simple model of spiking neurons," IEEE Transactions on neural networks, vol. 14, no. 6, pp. 1569-1572, 2003.

[4] V.M. Eguiluz, D.R. Chialvo, G.A. Cecchi, M. Baliki, and A.V. Apkarian, "Scale-free brain functional networks," Physical review letters, vol. 94, no. 1, p. 018102, 2005.

[5] D.H. Perkel, G.L. Gerstein, and G.P. Moore, "Neuronal spike trains and stochastic point processes: II simultaneous spike trains," Biophysical journal, vol. 7, no. 4, pp. 419-440, 1967.

[6] T. Schreiber, "Measuring information transfer," Physical review letters, vol. 85, no. 2, p. 461, 2000.

[7] F.V. Bussel, B. Kriener, and M. Timme, "Inferring synaptic connectivity from spatio-temporal spike patterns," Frontiers in computational neuroscience, vol. 5, p. 3, 2011.

[8] M. Timme, "Revealing network connectivity from response dynamics," Physical review letters, vol. 98, p. 224101, May 2007.

[9] Z. Levnajic and A. Pikovsky, "Network reconstruction from random phase resetting," Physical review letters, vol. 107, p. 034101, July 2011.

[10] S. Pajevic and D. Plenz, "Efficient network reconstruction from dynamical cascades identifies small-world topology of neuronal avalanches," PLoS computational biology, vol. 5, no. 1, p. e1000271, 2009.

[11] T. Leleu and K. Aihara, "Unambiguous reconstruction of network structure using avalanche dynamics," Physical Review E, vol. 91, no. 2, p. 022804, 2015.

[12] J.M. Beggs and D. Plenz, "Neuronal avalanches in neocortical circuits," Journal of neuroscience, vol. 23 , no. 35 , pp. 11167-11177, 2003.

[13] N. Friedman, S. Ito, B. Brinkman, M. Shimono, R. DeVille, K.A. Dahmen, J.M. Beggs, and T.C. Butler, "Universal critical dynamics in high resolution neuronal avalanche data," Physical review letters, vol. 108, no. 20, p. 208102, 2012.

[14] D.R. Chialvo, "Critical brain networks," Physica A: Statistical Mechanics and its Applications, vol. 340, no. 4, pp. 756-765, 2004. 
[15] A. Luczak, B.L. McNaughton, and K.D. Harris, "Packet-based communication in the cortex," Nature reviews. Neuroscience, vol. 16, no. 12, p. 745, 2015.

[16] M. Itoh and T. Leleu, "Modulation of context-dependent spatiotemporal patterns within packets of spiking activity," Neural Computation, vol. 29, no. 5, pp. 1263-1292, 2017.

[17] G. Hahn, and T. Petermann, M.N. Havenith, S. Yu, W. Singer, D. Plenz, and D. Nikolic, "Neuronal avalanches in spontaneous activity in vivo," Journal of neurophysiology, vol. 104, no. 6, pp. 3312-3322, 2010.

[18] E.D. Gireesh, and D. Plenz, "Neuronal avalanches organize as nested theta-and beta/gammaoscillations during development of cortical layer 2/3," PNAS, vol. 105, no. 21, pp. 7576-7581, 2008.

[19] Z. Nenadic and J.W. Burdick, "Spike detection using the continuous wavelet transform," IEEE Transactions on Biomedical Engineering, vol. 52, no. 1, pp. 74-87, 2005.

[20] J.F. Beche, S. Bonnet, T. Levi, R. Escola, A. Noca, G. Charvet, and R. Guillemaud, "Real-time adaptive discrimination threshold estimation for embedded neural signals detection," Proc. 4th International Conference on Neural Engineering 2009 IEEE/EMBS (NER'09), pp. 597-600, 2009.

[21] J.O. Muthmann, H. Amin, E. Sernagor, A. Maccione, D. Panas, L. Berdondini, U.S. Bhalla, and M.H. Hennig, "Spike detection for large neural populations using high density multielectrode arrays," Frontiers in neuroinformatics, vol. 9, p. 28, 2015.

[22] S. Dodel, J.M. Herrmann, and T. Geisel, "Functional connectivity by cross-correlation clustering," Neurocomputing, vol. 44, pp. 1065-1070, 2002.

[23] M. Ambroise, S. Buccelli, F. Grassia, A. Pirog, Y. Bornat, M. Chiappalone, and T. Levi, "Biomimetic neural network for modifying biological dynamics during hybrid experiments," Artificial Life and Robotics, vol. 22, no. 3, pp. 398-403, 2017.

[24] C.W. Eurich, J.M. Herrmann, and U.A. Ernst, "Finite-size effects of avalanche dynamics," Physical review E, vol. 66, no. 6, p. 066137, 2002.

[25] T. Kohno, M. Sekikawa, J. Li, T. Nanami, and K. Aihara, "Qualitative-modeling-based silicon neurons and their networks," Frontiers in neuroscience, vol. 10, p. 273, 2016.

[26] A.L. Hodgkin, and A.F. Huxley, "A quantitative description of membrane current and its application to conduction and excitation in nerve," The Journal of physiology, vol. 117, no. 4, pp. 500-544, 1952. 